\title{
Integral equation procedure based on tailored orthogonal functions for the $X Y$ spin fluid in an external magnetic field
}

\author{
F. Lado \\ Department of Physics, North Carolina State University, Raleigh, North Carolina 27695-8202, USA \\ E. Lomba \\ Instituto de Química Física Rocasolano, CSIC, Serrano 119, E-28006 Madrid, Spain \\ (Received 3 September 2007; published 12 October 2007)
}

\begin{abstract}
The classical $X Y$ model describes particles in three-dimensional space that carry magnetic moments or spins whose motion is restricted to rotations in a plane. Introduction of an external magnetic field lying in the same plane then generates a system that is anisotropic in the azimuthal angle $\varphi$. We use numerical simulations and integral equation techniques to study this system, producing in the latter case a formalism that is identical to that of the simpler isotropic version having no external field. The basis for this simplification is a generalization $\mathcal{E}_{m}(\varphi)$ of the ordinary exponential basis set $e^{i m \varphi}$ that restores orthogonality in the presence of the external field. We display results of sample calculations obtained with two integral equation closures, reference hypernettedchain and soft mean-spherical approximation, both coupled to the Lovett-Mou-Buff-Wertheim relation, along with results from the numerical simulations for comparison. Construction of the $\mathcal{E}_{m}(\varphi)$ is described in an Appendix.
\end{abstract}

DOI: 10.1103/PhysRevE.76.041502

PACS number(s): 61.20.Gy, 02.60.-x, 75.10.Hk, 75.50.Mm

\section{INTRODUCTION}

The response to internal and external magnetic fields of molecular magnetic moments in a classical fluid is variously modeled as occurring along an axis (Ising model), on a plane (XY model), or in space (Heisenberg model). All of these instances have long been studied using the basic techniques of equilibrium statistical mechanics: Mean field theory [1-7], integral equations [8-16], and numerical simulation $[4,7,11,16-19]$. In this work, we focus on one of the less studied cases, the $X Y$ spin fluid in an external magnetic field, with the purpose of extending to it the integral equation technique based on generalized orthogonal functions that we have previously used for the Heisenberg model $[10,11]$. We are following on the recent work of Omelyan et al. $[14,15]$, who have presented the first integral equation studies of this model. Academic though this model may appear, it turns out to be useful in the description of superfluid and demixing transitions in ${ }^{2} \mathrm{He}-{ }^{4} \mathrm{He}$ mixtures [20-22].

The classical $X Y$ spin fluid ( $N$ particles in volume $V$ at temperature $T$ ) in an external magnetic field $\mathbf{B}_{0}$ is defined by the canonical partition function

$$
\begin{aligned}
Z= & \frac{1}{N !\left(2 \pi \Lambda^{3}\right)^{N}} \int \prod_{j=1}^{N}\left(d \mathbf{r}_{j} d \varphi_{j}\right) \\
& \times \exp \left[\beta \sum_{j} \boldsymbol{\mu}_{j} \cdot \mathbf{B}_{0}-\beta \sum_{i<j} u_{0}\left(r_{i j}\right)-\beta \sum_{i<j} u_{s s}\left(r_{i j}, \varphi_{i}, \varphi_{j}\right)\right] .
\end{aligned}
$$

Here $\beta=1 / k_{B} T$ is the inverse Kelvin temperature, with $k_{B}$ Boltzmann's constant, $\Lambda$ the de Broglie thermal wavelength (which plays only a dimensional role), and $\varphi$ the orientation of a magnetic dipole moment $\boldsymbol{\mu}$ that is free to rotate in a plane, denoted the $x y$ plane. The uniform external magnetic field $\mathbf{B}_{0}$ also lies in that plane and its direction is taken to define the $x$ axis, so that $\varphi$ is the azimuthal angle and $\boldsymbol{\mu} \cdot \mathbf{B}_{0}=\mu B_{0} \cos \varphi, 0 \leqslant \varphi \leqslant 2 \pi$. The internal energy of the system is written as pairwise sums of a spherically symmetric potential $u_{0}(r)$ and a spin-spin potential $u_{s s}\left(r, \varphi_{1}, \varphi_{2}\right)$. We follow Omelyan et al. $[14,15]$ in putting

$$
u_{0}(r)=u_{0}^{\mathrm{rep}}(r)+u_{0}^{\mathrm{att}}(r)
$$

where $u_{0}^{\text {rep }}(r)$ is the repulsive part of the Lennard-Jones potential (truncated at its minimum and shifted),

$$
u_{0}^{\mathrm{rep}}(r)= \begin{cases}4 \epsilon\left[(\sigma / r)^{12}-(\sigma / r)^{6}\right]+\epsilon, & r<2^{1 / 6} \sigma, \\ 0, & r \geqslant 2^{1 / 6} \sigma,\end{cases}
$$

and $u_{0}^{\text {att }}(r)$ is an attractive potential of Yukawa form,

$$
\begin{gathered}
u_{0}^{\mathrm{att}}(r)=-\xi J(r), \\
J(r)=\frac{\epsilon}{r / \sigma} e^{-\kappa(r / \sigma-1)} .
\end{gathered}
$$

Here $\xi$ and $\kappa$ are dimensionless strength and range parameters, respectively. Finally, the spin-spin interaction tending to align two spins with one another is taken to be of $X Y$ form $[14,15]$,

$$
u_{s s}\left(r, \varphi_{1}, \varphi_{2}\right)=-J(r) \hat{\mathbf{s}}_{1} \cdot \hat{\mathbf{s}}_{2}=-J(r) \cos \varphi_{12},
$$

where $\hat{\mathbf{s}}$ is the unit spin vector in the direction of $\boldsymbol{\mu}$ and $\varphi_{12} \equiv \varphi_{1}-\varphi_{2}$.

The formal elements of the calculation are neatly displayed by factoring the partition function $Z=Z^{\text {id }} Z^{\text {ex }}$ into an ideal part and the excess; we get 


$$
\begin{gathered}
Z^{\mathrm{id}}=\frac{1}{N !\left(2 \pi \Lambda^{3}\right)^{N}} \int_{j=1}^{N}\left(d \mathbf{r}_{j} d \varphi_{j}\right) \exp \left[\beta \sum_{j} \boldsymbol{\mu}_{j} \cdot \mathbf{B}_{0}\right] \\
=\frac{1}{N !}\left[\frac{V I_{0}\left(\beta \mu B_{0}\right)}{\Lambda^{3}}\right]^{N} \\
Z^{\mathrm{ex}}=\frac{1}{(2 \pi V)^{N}} \int \prod_{j=1}^{N}\left[d \mathbf{r}_{j} d \varphi_{j} f_{0}\left(\varphi_{j}\right)\right] \\
\quad \times \exp \left[-\beta \sum_{i<j} u\left(r_{i j}, \varphi_{i}, \varphi_{j}\right)\right]
\end{gathered}
$$

where $u\left(r, \varphi_{1}, \varphi_{2}\right) \equiv u_{0}(r)+u_{s s}\left(r, \varphi_{1}, \varphi_{2}\right)$ is the total pair potential. This defines in Eq. (8) the one-body distribution function $f_{0}(\varphi)$ in the noninteracting $X Y$ spin system,

$$
f_{0}(\varphi)=\frac{e^{\beta \mu B_{0} \cos \varphi}}{I_{0}\left(\beta \mu B_{0}\right)},
$$

normalized such that

$$
\frac{1}{2 \pi} \int_{0}^{2 \pi} d \varphi f_{0}(\varphi)=1
$$

In these expressions, $I_{0}(z)$ is the modified Bessel function of order zero.

\section{ANISOTROPIC INTEGRAL EQUATIONS}

\section{A. One-body and two-body distribution functions}

Since the molecules are subject to both one-body and two-body forces, a complete statistical description of the system requires knowing both the one-body and two-body density functions,

$$
\begin{aligned}
& \rho^{(1)}(\mathbf{r}, \varphi)=\left\langle\sum_{j=1}^{N} \delta\left(\mathbf{r}-\mathbf{r}_{j}\right) \delta\left(\varphi-\varphi_{j}\right)\right\rangle=\frac{\rho}{2 \pi} f(\varphi), \\
& \rho^{(2)}\left(\mathbf{r}, \varphi, \mathbf{r}^{\prime}, \varphi^{\prime}\right) \\
& =\left\langle\sum_{i \neq j} \delta\left(\mathbf{r}-\mathbf{r}_{i}\right) \delta\left(\varphi-\varphi_{i}\right) \delta\left(\mathbf{r}^{\prime}-\mathbf{r}_{j}\right) \delta\left(\varphi^{\prime}-\varphi_{j}\right)\right\rangle \\
& =\frac{\rho^{2}}{(2 \pi)^{2}} f(\varphi) f\left(\varphi^{\prime}\right) g\left(\left|\mathbf{r}-\mathbf{r}^{\prime}\right|, \varphi, \varphi^{\prime}\right),
\end{aligned}
$$

where $\rho=N / V$ is the density and $f(\varphi)$ the one-body orientational distribution in the interacting fluid. Equation (12) defines the generalized pair distribution function $g\left(r, \varphi, \varphi^{\prime}\right)$ of the anisotropic spin system in an external magnetic field. The angular brackets in these definitions denote a canonical ensemble average exemplified in Eq. (1).

The basic equations that determine the distribution functions $f(\varphi)$ and $g\left(r, \varphi_{1}, \varphi_{2}\right)$ are well known [23,24]. The onebody distribution function $f(\varphi)$ can be calculated using the Lovett-Mou-Buff-Wertheim (LMBW) equation [25,26],

$$
\begin{aligned}
\frac{d}{d \varphi_{1}} \ln \left[\frac{f\left(\varphi_{1}\right)}{f_{0}\left(\varphi_{1}\right)}\right]= & \frac{\rho}{2 \pi} \int d \mathbf{r} \int_{0}^{2 \pi} d \varphi_{2} c\left(r, \varphi_{1}, \varphi_{2}\right) \frac{d f\left(\varphi_{2}\right)}{d \varphi_{2}} \\
= & \frac{\rho}{2 \pi} \int d \mathbf{r} \int_{0}^{2 \pi} d \varphi_{2} f\left(\varphi_{2}\right) c\left(r, \varphi_{1}, \varphi_{2}\right) \\
& \times \frac{d \ln f\left(\varphi_{2}\right)}{d \varphi_{2}},
\end{aligned}
$$

where $c\left(r, \varphi_{1}, \varphi_{2}\right)$ is the anisotropic direct correlation function (see below). Alternatively, the one-body density, Eq. (11), can be differentiated with respect to $\varphi$ to give the first member of a Kirkwood-Born-Green-Yvon (KBGY) hierarchy [23],

$$
\begin{aligned}
\frac{d}{d \varphi_{1}} \ln \left[\frac{f\left(\varphi_{1}\right)}{f_{0}\left(\varphi_{1}\right)}\right]= & -\frac{\rho}{2 \pi} \int d \mathbf{r} \int_{0}^{2 \pi} d \varphi_{2} f\left(\varphi_{2}\right) \\
& \times g\left(r, \varphi_{1}, \varphi_{2}\right) \frac{d \beta u_{s s}\left(r, \varphi_{1}, \varphi_{2}\right)}{d \varphi_{1}}
\end{aligned}
$$

Calculation of $f(\varphi)$ from these equations requires knowing the pair function $c\left(r, \varphi_{1}, \varphi_{2}\right)$ or $g\left(r, \varphi_{1}, \varphi_{2}\right)$. In classical liquid state theory, the pair distribution function is obtained from the Ornstein-Zernike (OZ) equation and a closure relation $[23,24]$. The first of these, generalized for anisotropy, reads

$$
\begin{aligned}
\gamma\left(r_{12}, \varphi_{1}, \varphi_{2}\right)= & \frac{\rho}{2 \pi} \int d \mathbf{r}_{3} d \varphi_{3} f\left(\varphi_{3}\right)\left[\gamma\left(r_{13}, \varphi_{1}, \varphi_{3}\right)\right. \\
& \left.+c\left(r_{13}, \varphi_{1}, \varphi_{3}\right)\right] c\left(r_{32}, \varphi_{3}, \varphi_{2}\right)
\end{aligned}
$$

for the indirect correlation function $\gamma=g-1-c$. The second, or closure, relation expresses the direct correlation function $c$ back in terms of $\gamma$ and the model's pair interactions,

$$
\begin{aligned}
c\left(r, \varphi_{1}, \varphi_{2}\right)= & \exp \left[-\beta u_{0}(r)-\beta u_{s s}\left(r, \varphi_{1}, \varphi_{2}\right)+\gamma\left(r, \varphi_{1}, \varphi_{2}\right)\right. \\
& \left.+b\left(r, \varphi_{1}, \varphi_{2}\right)\right]-1-\gamma\left(r, \varphi_{1}, \varphi_{2}\right) .
\end{aligned}
$$

This relation must be supplemented with an approximation for $b$, the so-called bridge function [27], which is formally defined in terms of a diagram summation [23] that offers little practical benefit. Most approximate closures for $c$ define $b$ implicitly.

For a given $f(\varphi)$, Eqs. (15) and (16) are solved iteratively for $\gamma\left(r, \varphi_{1}, \varphi_{2}\right)$ and $c\left(r, \varphi_{1}, \varphi_{2}\right)$, after which one can find

$$
\begin{aligned}
g\left(r, \varphi_{1}, \varphi_{2}\right)= & \exp \left[-\beta u_{0}(r)-\beta u_{s s}\left(r, \varphi_{1}, \varphi_{2}\right)+\gamma\left(r, \varphi_{1}, \varphi_{2}\right)\right. \\
& \left.+b\left(r, \varphi_{1}, \varphi_{2}\right)\right]
\end{aligned}
$$

and evaluate Eq. (13) or (14). (In the results reported below we have used the LMBW relation.) This produces a new $f(\varphi)$ and so requires a new round for $\gamma\left(r, \varphi_{1}, \varphi_{2}\right)$ and $c\left(r, \varphi_{1}, \varphi_{2}\right)$, which depend on this function through Eq. (15). These iterations continue until both one-body and two-body functions are self-consistently determined.

It is obvious that to actually carry out a numerical version of these procedures with Eqs. (13)-(16), the equations must first be simplified. 


\section{B. Tailored exponential functions}

Pair functions such as $\gamma\left(r, \varphi_{1}, \varphi_{2}\right)$ may be expanded in an angle-dependent basis set so that the orientational information they contain might be effectively conveyed by a small set of expansion coefficients. Thus one could write, for example,

$$
\gamma\left(r, \varphi_{1}, \varphi_{2}\right)=\sum_{m_{1}, m_{2}} \Gamma_{m_{1} m_{2}}(r) E_{m_{1}}\left(\varphi_{1}\right) E_{m_{2}}^{*}\left(\varphi_{2}\right),
$$

where the asterisk denotes complex conjugate and

$$
E_{m}(\varphi) \equiv e^{i m \varphi}=T_{m}(\cos \varphi)+i V_{m}(\cos \varphi) .
$$

Here $T_{m}(\cos \varphi)=\cos m \varphi$ is the type I Chebyshev polynomial of order $m$ and $V_{m}(\cos \varphi)=\sin m \varphi$ the associated Chebyshev function of order $m$ [28]. The familiar exponential functions $E_{m}(\varphi)$ of course have the orthonormalization

$$
\frac{1}{2 \pi} \int_{0}^{2 \pi} d \varphi E_{m}(\varphi) E_{m^{\prime}}^{*}(\varphi)=\delta_{m m^{\prime}}
$$

for $m, m^{\prime}$ positive or negative integers. We write the expansion coefficients in Eq. (18) in upper case $\Gamma$ to distinguish them from the distinct set of coefficients written in lower case $\gamma$ to be introduced next.

The external field $B_{0}$ along the $x$ axis produces a nonuniform distribution $f(\varphi)$ that enters the integrand of Eq. (20) and spoils the orthogonality of the standard exponential functions $E_{m}(\varphi)$. To regain the valuable property of orthogonality and work conveniently with the external field turned on, we introduce new basis functions $\mathcal{E}_{m}(\varphi)$ that are orthonormal with weight function $f(\varphi)$,

$$
\frac{1}{2 \pi} \int_{0}^{2 \pi} d \varphi f(\varphi) \mathcal{E}_{m}(\varphi) \mathcal{E}_{m^{\prime}}^{*}(\varphi)=\delta_{m m^{\prime}}
$$

again for $m, m^{\prime}$ positive or negative integers, where

$$
\begin{gathered}
\mathcal{E}_{m}(\varphi)=\mathcal{T}_{m}(\cos \varphi)+i \mathcal{V}_{m}(\cos \varphi), \\
\mathcal{E}_{-m}(\varphi)=\mathcal{E}_{m}^{*}(\varphi),
\end{gathered}
$$

for $m \geqslant 0$. Construction of the needed generalized Chebyshev functions $\mathcal{T}_{m}(\cos \varphi)$ and $\mathcal{V}_{m}(\cos \varphi)$ is described in Appendix A.

Equation (18) is now generalized to read

$$
\gamma\left(r, \varphi_{1}, \varphi_{2}\right)=\sum_{m_{1}, m_{2}} \gamma_{m_{1} m_{2}}(r) \mathcal{E}_{m_{1}}\left(\varphi_{1}\right) \mathcal{E}_{m_{2}}^{*}\left(\varphi_{2}\right)
$$

In Eq. (24), $m_{1}$ and $m_{2}$ range as noted over positive and negative integers. With Eq. (21), its inversion becomes

$$
\begin{aligned}
\gamma_{m_{1} m_{2}}(r)= & \frac{1}{(2 \pi)^{2}} \int_{0}^{2 \pi} d \varphi_{1} \int_{0}^{2 \pi} d \varphi_{2} f\left(\varphi_{1}\right) f\left(\varphi_{2}\right) \\
& \times \gamma\left(r, \varphi_{1}, \varphi_{2}\right) \mathcal{E}_{m_{1}}^{*}\left(\varphi_{1}\right) \mathcal{E}_{m_{2}}\left(\varphi_{2}\right) .
\end{aligned}
$$

Coefficients of all pair functions satisfy the symmetries

$$
\gamma_{m_{1} m_{2}}(r)=\gamma_{m_{2} m_{1}}(r)=\gamma_{\bar{m}_{2} \bar{m}_{1}}(r)=\gamma_{\bar{m}_{1} \bar{m}_{2}}(r),
$$

where $\bar{m} \equiv-m$.
A specific expansion needed in the sequel is that of the spin-spin interaction, Eq. (6), where we have

$$
\cos \varphi_{12}=\cos \varphi_{1} \cos \varphi_{2}+\sin \varphi_{1} \sin \varphi_{2},
$$

while, from Appendix A,

$$
\begin{gathered}
\cos \varphi=\nu_{1}+\frac{1}{2}\left(1-2 \nu_{1}^{2}+\nu_{2}\right)^{1 / 2}\left[\mathcal{E}_{1}(\varphi)+\mathcal{E}_{1}^{*}(\varphi)\right] \\
\sin \varphi=\frac{1}{2 i}\left(1-\nu_{2}\right)^{1 / 2}\left[\mathcal{E}_{1}(\varphi)-\mathcal{E}_{1}^{*}(\varphi)\right]
\end{gathered}
$$

Here the $\nu_{k}$ are quasimoments of $f(\varphi)$, defined in Eq. (A2). Combining these expressions and simplifying, we find

$$
u_{s s}\left(r, \varphi_{1}, \varphi_{2}\right)=-J(r) \sum_{m_{1}, m_{2}} C_{m_{1} m_{2}} \mathcal{E}_{m_{1}}\left(\varphi_{1}\right) \mathcal{E}_{m_{2}}^{*}\left(\varphi_{2}\right)
$$

with nonzero coefficients

$$
\begin{gathered}
C_{00}=\nu_{1}^{2}, \\
C_{1-1}=C_{-11}=\frac{1}{2}\left(\nu_{2}-\nu_{1}^{2}\right), \\
C_{10}=C_{01}=C_{0-1}=C_{-10}=\frac{1}{2} \nu_{1}\left(1-2 \nu_{1}^{2}+\nu_{2}\right)^{1 / 2}, \\
C_{11}=C_{-1-1}=\frac{1}{2}\left(1-\nu_{1}^{2}\right) .
\end{gathered}
$$

\section{Ornstein-Zernike equation}

Numerical evaluation of the Ornstein-Zernike equation is simpler in Fourier transform representation, which deconvolves the direct space integral. In transform space, Eq. (15) becomes

$$
\begin{aligned}
\tilde{\gamma}\left(k, \varphi_{1}, \varphi_{2}\right)= & \frac{\rho}{2 \pi} \int_{0}^{2 \pi} d \varphi_{3} f\left(\varphi_{3}\right)\left[\widetilde{\gamma}\left(k, \varphi_{1}, \varphi_{3}\right)\right. \\
& \left.+\widetilde{c}\left(k, \varphi_{1}, \varphi_{3}\right)\right] \widetilde{c}\left(k, \varphi_{3}, \varphi_{2}\right),
\end{aligned}
$$

with one remaining integration. Because the orientations of spins 1 and 2 and that of $\mathbf{r}_{12}$ are decoupled in the $X Y$ model, the transforms may be performed holding the former fixed. A transform pair is then

$$
\begin{gathered}
\tilde{\gamma}\left(k, \varphi_{1}, \varphi_{2}\right)=4 \pi \int_{0}^{\infty} d r r^{2} \gamma\left(r, \varphi_{1}, \varphi_{2}\right) \frac{\sin (k r)}{k r}, \\
\gamma\left(r, \varphi_{1}, \varphi_{2}\right)=\frac{1}{2 \pi^{2}} \int_{0}^{\infty} d k k^{2} \tilde{\gamma}\left(k, \varphi_{1}, \varphi_{2}\right) \frac{\sin (k r)}{k r}
\end{gathered}
$$

and the transformed functions may themselves be expanded,

$$
\tilde{\gamma}\left(k, \varphi_{1}, \varphi_{2}\right)=\sum_{m_{1}, m_{2}} \tilde{\gamma}_{m_{1} m_{2}}(k) \mathcal{E}_{m_{1}}\left(\varphi_{1}\right) \mathcal{E}_{m_{2}}^{*}\left(\varphi_{2}\right),
$$

with expansion coefficients $\tilde{\gamma}_{m_{1} m_{2}}(k)$ that are directly the Fourier transforms of the corresponding $\gamma_{m_{1} m_{2}}(r)$. 
We may now begin to garner the profit from the special orthogonality built into the basis set $\mathcal{E}_{m}(\varphi)$. Expanding the transforms as in Eq. (35), we find the final integral in Eq. (32) can be carried out to yield a relation between expansion coefficients alone,

$$
\widetilde{\gamma}_{m_{1} m_{2}}(k)=\rho \sum_{m_{3}}\left[\widetilde{\gamma}_{m_{1} m_{3}}(k)+\widetilde{c}_{m_{1} m_{3}}(k)\right] \widetilde{c}_{m_{3} m_{2}}(k),
$$

which can be solved for the $\widetilde{\gamma}_{m_{1} m_{2}}(k)$; in matrix form,

$$
\widetilde{\boldsymbol{\Gamma}}(k)=\rho \widetilde{\mathbf{C}}(k) \widetilde{\mathbf{C}}(k)[\mathbf{I}-\rho \widetilde{\mathbf{C}}(k)]^{-1},
$$

where $\widetilde{\boldsymbol{\Gamma}}(k)$ and $\widetilde{\mathbf{C}}(k)$ are symmetric matrices with elements $\widetilde{\gamma}_{m_{1} m_{2}}(k)$ and $\widetilde{c}_{m_{1} m_{2}}(k)$, respectively, and $\mathbf{I}$ is the unit matrix. The notable feature of Eq. (37) is that it is identical to that of an isotropic system with $B_{0}=0$. All of the anisotropy is built into the basis functions.

The closure relation (16) is also reduced to manipulation of coefficients. For a given $r$, first the full function $g\left(r, \varphi_{1}, \varphi_{2}\right)$ is assembled in Eq. (17) by performing summations like Eq. (24) for the terms in its exponent; this is followed by calculation of its coefficients $g_{m_{1} m_{2}}(r)$ with an inversion like that of Eq. (25). Then the $c_{m_{1} m_{2}}(r)=g_{m_{1} m_{2}}(r)$ $-\delta_{m_{1} 0} \delta_{m_{2} 0}-\gamma_{m_{1} m_{2}}(r)$ follow from Eq. (16) in coefficient form.

One cycle of the iterative (numerical) solution that yields a converged set of coefficients $\left\{\gamma_{m_{1} m_{2}}(r)\right\}$ will then consist of four steps:

$$
\begin{aligned}
& \left\{\gamma_{m_{1} m_{2}}(r)\right\} \stackrel{\text { closure }}{\longrightarrow}\left\{c_{m_{1} m_{2}}(r)\right\} \stackrel{(\mathrm{FT})}{\longrightarrow}\left\{\widetilde{c}_{m_{1} m_{2}}(k)\right\} \\
& \quad \stackrel{\mathrm{OZ}}{\rightarrow}\left\{\widetilde{\gamma}_{m_{1} m_{2}}(k)\right\} \stackrel{(\mathrm{FT})^{-1}}{\longrightarrow}\left\{\gamma_{m_{1} m_{2}}(r)\right\} .
\end{aligned}
$$

\section{LMBW equation for $f(\varphi)$}

Equation (13) also simplifies smartly with expansions in the generalized Chebyshev functions. We put

$$
\ln f(\varphi)=\sum_{m=0}^{\infty} A_{m} \mathcal{T}_{m}(\cos \varphi)
$$

and seek to find the $A_{m}$. (Actually, $A_{0}$ is determined by normalization, so we are concerned with $\left.A_{1}, A_{2}, \ldots\right)$ Expanding $c\left(r, \varphi_{1}, \varphi_{2}\right)$, we first find from Eq. (13) that

$$
\begin{aligned}
\sum_{m_{2}=1}^{\infty} A_{m_{2}} \frac{d \mathcal{T}_{m_{2}}\left(\cos \varphi_{1}\right)}{d \varphi_{1}}= & \beta \mu B_{0} \frac{d \cos \varphi_{1}}{d \varphi_{1}} \\
& +\sum_{m_{1}, m_{2}=1}^{\infty} \sum_{m_{3}=-\infty}^{\infty} \mathcal{E}_{m_{1}}\left(\varphi_{1}\right) \rho \widetilde{c}_{m_{1} m_{3}}(0) \\
& \times i D_{m_{3} m_{2}} A_{m_{2}},
\end{aligned}
$$

where

$$
D_{m_{1} m_{2}} \equiv \frac{-i}{2 \pi} \int_{0}^{2 \pi} d \varphi f(\varphi) \mathcal{E}_{m_{1}}^{*}(\varphi) \frac{d \mathcal{T}_{m_{2}}(\cos \varphi)}{d \varphi} .
$$

It follows then that

$$
\begin{aligned}
\sum_{m_{2}=1}^{\infty} D_{m_{1} m_{2}} A_{m_{2}}= & \frac{1}{2} \beta \mu B_{0}\left(1-\nu_{2}\right)^{1 / 2} \delta_{m_{1} 1} \\
& +\sum_{m_{2}=1}^{\infty} \sum_{m_{3}=-\infty}^{\infty} \rho \widetilde{c}_{m_{1} m_{3}}(0) D_{m_{3} m_{2}} A_{m_{2}},
\end{aligned}
$$

or more briefly

$$
\sum_{m_{2}=1}^{\infty} Q_{m_{1} m_{2}} A_{m_{2}}=\frac{1}{2} \beta \mu B_{0}\left(1-\nu_{2}\right)^{1 / 2} \delta_{m_{1} 1},
$$

where

$$
Q_{m_{1} m_{2}} \equiv \sum_{m_{3}=-\infty}^{\infty}\left[\delta_{m_{1} m_{3}}-\rho \widetilde{c}_{m_{1} m_{3}}(0)\right] D_{m_{3} m_{2}} .
$$

For $m_{1}, m_{2}>0$, the matrix elements $D_{m_{1} m_{2}}$ are nonzero only for $m_{2}=m_{1}, m_{1}+1$, while $D_{\bar{m}_{1} m_{2}}=-D_{m_{1} m_{2}}$. We have finally from Eq. (43),

$$
A_{m}=\frac{1}{2} \beta \mu B_{0}\left(1-\nu_{2}\right)^{1 / 2} R_{m 1},
$$

where in matrix notation $\mathbf{R} \equiv \mathbf{Q}^{-1}$.

If it happens that $A_{1}$ is much larger than $A_{2}, A_{3}, \ldots$, then we may ignore these higher coefficients in the analysis above to get

$$
\begin{aligned}
A_{1} \approx & \frac{\beta \mu B_{0}\left(1-\nu_{2}\right)^{1 / 2}}{2 D_{11}\left[1-\rho \widetilde{c}_{11}(0)+\rho \widetilde{c}_{1-1}(0)\right]}, \\
& \approx \frac{\beta \mu B_{0}\left(1-2 \nu_{1}^{2}+\nu_{2}\right)^{1 / 2}}{1-\rho \widetilde{c}_{11}(0)+\rho \widetilde{c}_{1-1}(0)},
\end{aligned}
$$

where we have used $D_{11}=\frac{1}{2}\left[\left(1-\nu_{2}\right) /\left(1-2 \nu_{1}^{2}+\nu_{2}\right)\right]^{1 / 2}$. Then the form of the angular distribution function remains unchanged,

$$
\ln f(\varphi) \approx \mathrm{const}+\beta \mu B \cos \varphi,
$$

but the effective field in the interacting system becomes

$$
B=\frac{B_{0}}{1-\rho \widetilde{c}_{11}(0)+\rho \widetilde{c}_{1-1}(0)} .
$$

We will find that Eqs. (47) and (48) are effectively exact in the context of the numerical solutions. We further anticipate the calculations of Sec. IV by noting that the alternative approach based on the KBGY relation, Eq. (14), although not further discussed here, produces essentially the same effective fields.

\section{THERMODYNAMICS}

Once the one-body and two-body distribution functions have been determined, it is straightforward to calculate the various thermodynamic quantities. For instance, the internal energy $E=-(\partial \ln Z / \partial \beta)$ and the pressure $p=k_{B} T(\partial \ln Z / \partial V)$ are found as quadratures, 


$$
\begin{gathered}
\frac{\beta E}{N}=\frac{3}{2}-\beta \mu B_{0} \nu_{1}+\frac{1}{2} \rho \int d \mathbf{r} g_{00}(r) \beta u_{0}(r) \\
+\frac{1}{2} \rho \int d \mathbf{r} \sum_{m_{1}, m_{2}} g_{m_{1} m_{2}}(r) \beta u_{m_{1} m_{2}}^{(s s)}(r), \\
\frac{\beta p}{\rho}=1-\frac{1}{6} \rho \int d \mathbf{r} g_{00}(r) r \frac{d \beta u_{0}(r)}{d r}+\frac{1}{6} \rho \int d \mathbf{r}\left(1+\kappa \frac{r}{\sigma}\right) \\
\times \sum_{m_{1}, m_{2}} g_{m_{1} m_{2}}(r) \beta u_{m_{1} m_{2}}^{(s s)}(r),
\end{gathered}
$$

in terms of the coefficients of the distribution function $g\left(r, \varphi_{1}, \varphi_{2}\right)$ and the pair potentials $u_{0}(r)$ and $u_{s s}\left(r, \varphi_{1}, \varphi_{2}\right)$, while the isothermal compressibility $K_{T}$ is given by

$$
\rho k_{B} T K_{T}=1+\rho \widetilde{h}_{00}(0),
$$

where $h=g-1$. Finally, an expression for the Helmholtz free energy is presented in Appendix B. One notes again that expansions in the generalized basis set $\mathcal{E}_{m}(\varphi)$ lead to equations that are identical to those of an isotropic system.

Further, we can compute the magnetic properties of the $X Y$ spin fluid in a uniform magnetic field $\mathbf{B}_{0}$. The net magnetization in the direction $\alpha=x, y$ is

$$
M_{\alpha}=\frac{1}{\beta} \frac{\partial \ln Z}{\partial B_{0 \alpha}}=\mu\left\langle\sum_{j} s_{j \alpha}\right\rangle,
$$

and so the magnetic susceptibility components are

$$
\begin{aligned}
\chi_{\alpha \beta} \equiv & \frac{1}{V} \frac{\partial M_{\alpha}}{\partial B_{0 \beta}}=\frac{\beta \mu^{2}}{V}\left[\left\langle\left(\sum_{i} s_{i \alpha}\right)\left(\sum_{j} s_{j \beta}\right)\right\rangle-\left\langle\sum_{i} s_{i \alpha}\right\rangle\right. \\
& \left.\times\left\langle\sum_{j} s_{j \beta}\right\rangle\right] .
\end{aligned}
$$

Now with $\mathbf{B}_{0}$ defining the $x$ axis, we get the longitudinal, $\chi_{L} \equiv \chi_{x x}$, and transverse, $\chi_{T} \equiv \chi_{y y}$, magnetic susceptibilities as

$$
\begin{aligned}
\chi_{L} / \beta \rho \mu^{2}= & \nu_{1}^{2}\left[1+\rho \widetilde{h}_{00}(0)\right]+2 \nu_{1}\left(1-2 \nu_{1}^{2}+\nu_{2}\right)^{1 / 2} \rho \widetilde{h}_{10}(0) \\
& +\frac{1}{2}\left(1-2 \nu_{1}^{2}+\nu_{2}\right)\left[1+\rho \widetilde{h}_{11}(0)+\rho \widetilde{h}_{1-1}(0)\right],
\end{aligned}
$$

$$
\chi_{T} / \beta \rho \mu^{2}=\frac{1}{2}\left(1-\nu_{2}\right)\left[1+\rho \tilde{h}_{11}(0)-\rho \tilde{h}_{1-1}(0)\right] .
$$

The off-diagonal elements of $\boldsymbol{\chi}$ vanish, while the magnetization is $M=N \mu \nu_{1}$.

\section{SAMPLE RESULTS}

A very thorough integral equation study of the $X Y$ spin fluid in an external magnetic field has recently been published by Omelyan et al. [15] using a soft mean-spherical approximation (SMSA) and we need not duplicate that scope of data. That work was based on expansions in the ordinary Chebyshev functions $T_{m}(\cos \varphi)=\cos m \varphi$ and $V_{m}(\cos \varphi)$ $=\sin m \varphi$. Here we aim to present just a small sample of additional data to illustrate application of the alternative approach using orthogonal basis functions $\mathcal{T}_{m}(\cos \varphi)$ and $\mathcal{V}_{m}(\cos \varphi)$ that are specifically tailored to the anisotropic environment created by the external field; aside from the enhanced simplicity of the formal expressions already manifest in the equations displayed above, the most notable difference is found in the results for $f(\varphi)$.

The interaction of an individual spin with the external field involves the familiar dipole potential, $\boldsymbol{- \mu} \cdot \mathbf{B}_{0}$, and leads to the unperturbed distribution function

$$
f_{0}(\varphi)=\frac{e^{\beta \mu B_{0} \cos \varphi}}{I_{0}\left(\beta \mu B_{0}\right)} .
$$

For the interacting system, Omelyan et al. [15] find that in setting

$$
\ln f(\varphi)=\mathrm{const}+\beta \mu B_{0} \cos \varphi+\beta \sum_{m=1}^{\infty} a_{m} T_{m}(\cos \varphi),
$$

with standard Chebyshev polynomials $T_{m}(\cos \varphi), a_{2}$ can be as large as $30 \%$ of $a_{1}$ and only terms $a_{m}$ with $m>3$ are negligible. In contrast, we find that in setting

$$
\ln f(\varphi)=\sum_{m=0}^{\infty} A_{m} \mathcal{T}_{m}(\cos \varphi)
$$

with tailored Chebyshev polynomials $\mathcal{T}_{m}(\cos \varphi)$, no more than the linear term in the expansion is significant; the coefficients of higher terms $\left(A_{2}, A_{3}, \ldots\right)$ are smaller than $A_{1}$ by two or more orders of magnitude. Correspondingly, the approximate $A_{1}$ of Eq. (46) differs from the exact $A_{1}$ of Eq. (45) by no more than $0.1 \%$; within the overall precision of a numerical calculation, Eq. (46) may be treated as exact. Thus $f(\varphi)$ retains the same functional form as the unperturbed system,

$$
f(\varphi)=\frac{e^{\beta \mu B \cos \varphi}}{I_{0}(\beta \mu B)},
$$

but with an effective field $B$ given by Eq. (48).

For the sample calculations here, we use both the SMSA and the reference hypernetted-chain (RHNC) closures, the latter optimized to achieve a minimum in the free energy.

The SMSA closure [23] is a generalization of the meanspherical approximation that is widely used for potentials with a hard core. Splitting the total potential $u\left(r, \varphi_{1}, \varphi_{2}\right)$ $=u^{\mathrm{SR}}\left(r, \varphi_{1}, \varphi_{2}\right)+u^{\mathrm{LR}}\left(r, \varphi_{1}, \varphi_{2}\right)$ into short-range (SR) and long-range (LR) parts, one replaces the formally exact but uncomputable [because of the unknown $\left.b\left(r, \varphi_{1}, \varphi_{2}\right)\right]$ Eq. (17) with the approximation

$$
\begin{aligned}
g^{\mathrm{SMSA}}\left(r, \varphi_{1}, \varphi_{2}\right)= & e^{-\beta u^{\mathrm{SR}}\left(r, \varphi_{1}, \varphi_{2}\right)}\left[1+\gamma\left(r, \varphi_{1}, \varphi_{2}\right)\right. \\
& \left.-\beta u^{\mathrm{LR}}\left(r, \varphi_{1}, \varphi_{2}\right)\right] .
\end{aligned}
$$

A particularly convenient choice for the potential splitting which we shall adopt here is 


$$
\begin{aligned}
g^{\mathrm{SMSA}}\left(r, \varphi_{1}, \varphi_{2}\right)= & e^{-\beta u_{0}^{\mathrm{rep}}(r)}\left[1+\gamma\left(r, \varphi_{1}, \varphi_{2}\right)-\beta u_{0}^{\text {att }}(r)\right. \\
& \left.-\beta u_{\mathrm{Ss}}\left(r, \varphi_{1}, \varphi_{2}\right)\right],
\end{aligned}
$$

since this linearizes the angular dependence of the pair distribution function. Its coefficients can then be immediately obtained by inspection,

$$
g_{00}^{\mathrm{SMSA}}(r)=e^{-\beta u_{0}^{\mathrm{rep}}(r)}\left[1+\gamma_{00}(r)-\beta u_{0}^{\mathrm{att}}(r)-\beta u_{00}^{(\mathrm{Ss})}(r)\right],
$$

$g_{m_{1} m_{2}}^{\mathrm{SMSA}}(r)=e^{-\beta u_{0}^{\mathrm{rep}}(r)}\left[\gamma_{m_{1} m_{2}}(r)-\beta u_{m_{1} m_{2}}^{(\mathrm{ss})}(r)\right], \quad$ for $m_{1} m_{2} \neq 00$,

which saves some computation time in the iteration step $\left\{\gamma_{m_{1} m_{2}}(r)\right\} \stackrel{\text { closure }}{\rightarrow}\left\{c_{m_{1} m_{2}}(r)\right\}$ compared to the RHNC procedure. (It should be noted that the full RHNC calculation is already extremely fast on any current personal computer-on the order of seconds per thermodynamic state.) If $u_{0}^{\text {rep }}(r)$ were the hard sphere potential, these equations would describe the standard mean-spherical approximation [23].

The RHNC closure $[29,30]$ consists in replacing the unknown function $b\left(r, \varphi_{1}, \varphi_{2}\right)$ in Eq. (17) with the bridge function $b^{\text {ref }}\left(r, \varphi_{1}, \varphi_{2}\right)$ obtained from some calculable reference model,

$$
\begin{aligned}
g^{\mathrm{RHNC}}\left(r, \varphi_{1}, \varphi_{2}\right)= & \exp \left[-\beta u_{0}(r)-\beta u_{s s}\left(r, \varphi_{1}, \varphi_{2}\right)+\gamma\left(r, \varphi_{1}, \varphi_{2}\right)\right. \\
& \left.+b^{\mathrm{ref}}\left(r, \varphi_{1}, \varphi_{2}\right)\right] .
\end{aligned}
$$

For convenience, in the present calculation we have chosen as reference a hard sphere fluid at the same density,

$$
b^{\text {ref }}\left(r, \varphi_{1}, \varphi_{2}\right) \approx b_{\mathrm{HS}}\left(r ; \sigma_{0}\right),
$$

where $\sigma_{0}$ is the reference sphere diameter that is varied to attain a minimized free energy [31]. For the modeled hard sphere functions we use the parametrizations of Verlet-Weis [32] and Henderson-Grundke [33].

In the RHNC calculation, expansions in $m$ such as Eqs. (24) and (39) have been extended through $|m|=4$; reduction to a maximum of $|m|=3$ yields unchanged results. For SMSA, the linearization of the closure in Eq. (61) limits the nonvanishing terms in all expansions to just those in the potential; i.e., through $|m|=1$. We note that given the symmetries (26), the number of distinct coefficients in the expansion of a pair function is $\left(m_{\max }+1\right)^{2}$ for a (maximum $|m|$ $\left.\equiv m_{\max }\right)=0,1,2,3,4, \ldots$.

We have set $\xi=\kappa=1$ in the pair potentials for all calculations reported [see Eqs. (4) and (5)]. Transform functions of $r$ and $k$ are connected through the fast Fourier transform [34] and are evaluated on uniform grids $r_{j}=j \Delta r, k_{j}=j \Delta k, j$ $=0,1,2, \ldots, N_{r}$, with $\Delta r \Delta k=\pi / N_{r}$ and $N_{r}=1024, \quad \Delta r$ $=0.02 \sigma$. Integrals over $r$ and $k$ for thermodynamic quantities are calculated with the trapezoidal rule using the same grid points, while Gauss-Chebyshev quadratures over angle, Eq. (A10), are carried out with $n \geqslant 10$ Gaussian root points.

With regard to the simulation, we have used a standard Monte Carlo method whose only peculiarity is the implementation of orientational single-particle and cluster moves as described in Ref. [35], with the orientational degrees of freedom restricted to lie in the $x y$ plane. We have used a sample of 864 particles and performed averages over 50000 configurations. Each configuration implies 864 displacement attempts, with an acceptance ratio of 50\%, and either 864 single-particle moves or one cluster move. The initial 20000 configurations in each run were discarded to attain thermal equilibrium.

Thermodynamic and magnetic properties of the $X Y$ spin fluid computed with an RHNC-LMBW combination are presented in Table I. Additionally, in Table II the results obtained using the SMSA-LMBW combination are laid out for just the case of nonzero field. A global assessment of the quality of the closure approximations can be made from inspection of Fig. 1, in which these results are compared with Monte Carlo simulation data. From this figure, it is clear that the RHNC-LMBW approach is practically exact in the zero field case. At $\mu B_{0} / \varepsilon=5$, thermodynamic properties from RHNC-LMBW compare well with simulation data and the approach is clearly superior to the SMSA-LMBW. Small discrepancies show up as density increases, especially for the net magnetization $M / N \mu=\nu_{1}$. Interestingly, the simulation data for this quantity are bracketed by the SMSA-LMBW and RHNC-LMBW results. This points to a possible route to improve the quality of the theoretical approach. In the spirit of the Zerah-Hansen HMSA approximation [36], one might use a continuous interpolation scheme between the SMSA and HNC (or RHNC) closures to implement a thermodynamically self-consistent approach based on the net magnetization and the longitudinal susceptibility as the key quantities to check for consistency.

To illustrate the structure, we define the functions

$$
\begin{gathered}
g^{00}(r) \equiv\left\langle g\left(r, \varphi_{1}, \varphi_{2}\right)\right\rangle_{\varphi_{1} \varphi_{2}}=g_{00}(r), \\
g^{11}(r) \equiv\left\langle g\left(r, \varphi_{1}, \varphi_{2}\right) \cos \varphi_{12}\right\rangle_{\varphi_{1} \varphi_{2}}=\sum_{m_{1}, m_{2}} C_{m_{1} m_{2}} g_{m_{1} m_{2}}(r),
\end{gathered}
$$

where the averages are calculated as

$$
\begin{aligned}
\left\langle H\left(r, \varphi_{1}, \varphi_{2}\right)\right\rangle_{\varphi_{1} \varphi_{2}} \equiv & \frac{1}{(2 \pi)^{2}} \int_{0}^{2 \pi} d \varphi_{1} \int_{0}^{2 \pi} d \varphi_{2} \\
& \times f\left(\varphi_{1}\right) f\left(\varphi_{2}\right) H\left(r, \varphi_{1}, \varphi_{2}\right) .
\end{aligned}
$$

Plots of $g^{00}(r)$ and $g^{11}(r)$ for various representative states are shown in Figs. 2 and 3. The radial coefficient $g^{00}(r)$ is very well reproduced by both approximations; departures in the long-range behavior of $g^{11}(r)$ become apparent at the larger density and nonzero field. This is an immediate consequence of the deviations observed in the calculated magnetization, since

$$
\lim _{r \rightarrow \infty} g^{11}(r)=(M / N \mu)^{2} .
$$

Again, we see that the angular coefficient from the simulation lies within the boundaries formed by the SMSA-LMBW and RHNC-LMBW results. 
TABLE I. Thermodynamic and magnetic properties of the $X Y$ spin fluid in an external magnetic field $B_{0}$ at reduced temperature $k_{B} T / \epsilon=10$, calculated using the optimized RHNC closure with the LMBW equation; $\sigma_{0}$ is the optimized sphere diameter of the reference hard sphere system. The excess free energy is defined here as $\beta A^{\mathrm{ex}} / N \equiv \beta A / N-\ln \left(\rho \Lambda^{3}\right)+1$.

\begin{tabular}{ccccccccccc}
\hline \hline$\rho \sigma^{3}$ & $\sigma_{0} / \sigma$ & $\mu B_{0} / \epsilon$ & $\mu B / \epsilon$ & $\beta E / N-3 / 2$ & $\beta p / \rho-1$ & $\left(\rho k_{B} T K_{T}\right)^{-1}$ & $M / N \mu$ & $\chi_{L} / \rho \beta \mu^{2}$ & $\chi_{T} / \rho \beta \mu^{2}$ & $\beta A^{\mathrm{ex}} / N$ \\
\hline 0.10 & 0.8889 & 0 & 0 & -0.105 & 0.041 & 1.098 & 0 & 0.578 & 0.578 & 0.034 \\
0.20 & 0.8917 & 0 & 0 & -0.207 & 0.115 & 1.302 & 0 & 0.687 & 0.687 & 0.083 \\
0.30 & 0.8935 & 0 & 0 & -0.304 & 0.231 & 1.654 & 0 & 0.848 & 0.848 & 0.150 \\
0.40 & 0.8943 & 0 & 0 & -0.394 & 0.401 & 2.201 & 0 & 1.110 & 1.110 & 0.238 \\
0.50 & 0.8942 & 0 & 0 & -0.475 & 0.638 & 3.006 & 0 & 1.610 & 1.610 & 0.350 \\
0.60 & 0.8930 & 0 & 0 & -0.545 & 0.960 & 4.143 & 0 & 2.928 & 2.928 & 0.492 \\
0.70 & 0.8914 & 0 & 0 & -0.604 & 1.383 & 5.642 & 0 & 14.07 & 14.07 & 0.666 \\
0.10 & 0.8873 & 5 & 5.749 & -0.254 & 0.031 & 1.074 & 0.276 & 0.596 & 0.552 & -0.037 \\
0.20 & 0.8900 & 5 & 6.738 & -0.394 & 0.087 & 1.229 & 0.319 & 0.693 & 0.638 & 0.000 \\
0.30 & 0.8917 & 5 & 8.069 & -0.547 & 0.173 & 1.479 & 0.374 & 0.799 & 0.748 & 0.051 \\
0.40 & 0.8924 & 5 & 9.858 & -0.719 & 0.292 & 1.835 & 0.441 & 0.883 & 0.883 & 0.116 \\
0.50 & 0.8923 & 5 & 12.174 & -0.914 & 0.448 & 2.337 & 0.518 & 0.886 & 1.036 & 0.196 \\
0.60 & 0.8915 & 5 & 14.956 & -1.127 & 0.656 & 3.096 & 0.595 & 0.772 & 1.189 & 0.293 \\
0.70 & 0.8900 & 5 & 18.032 & -1.342 & 0.944 & 4.282 & 0.663 & 0.592 & 1.324 & 0.411 \\
0.80 & 0.8877 & 5 & 21.238 & -1.542 & 1.349 & 6.064 & 0.717 & 0.424 & 1.432 & 0.556 \\
0.90 & 0.8851 & 5 & 24.483 & -1.714 & 1.906 & 8.613 & 0.759 & 0.300 & 1.516 & 0.738 \\
1.00 & 0.8819 & 5 & 27.730 & -1.848 & 2.656 & 12.116 & 0.792 & 0.214 & 1.581 & 0.967 \\
\hline \hline
\end{tabular}

\section{CONCLUSIONS}

In summary, we have shown that a general integral equation procedure based on tailored orthogonal basis functions, successfully applied in previous works $[10,11]$ to describe the Heisenberg spin fluid in an external field, can be readily extended to the classical $X Y$ model in an external field. The specific RHNC-LMBW approximation is found to work reasonably well, although some deviations are noticed at high densities and nonzero fields. We speculate that the quality of the theoretical approximation might be improved by implementing self-consistency at the level of the magnetic quantities (i.e., magnetic susceptibility vs. magnetization).

\section{ACKNOWLEDGMENTS}

E.L. gratefully acknowledges financial support from the Dirección General de Investigación Científica y Técnica under Grant No. FIS2004-02954-C03-01 and the Dirección General de Universidades e Investigación de la Comunidad de Madrid under Grant No. S0505/ESP/0299, Program MOSSNOHO-CM.

\section{APPENDIX A: GENERALIZED CHEBYSHEV FUNCTIONS}

In principle, the needed generalization of the type I Chebyshev polynomials $T_{m}(\cos \varphi)=\cos m \varphi$ can be produced

TABLE II. Thermodynamic and magnetic properties of the $X Y$ spin fluid in an external magnetic field $B_{0}$ at reduced temperature $k_{B} T / \epsilon=10$, calculated using the SMSA closure with the LMBW equation.

\begin{tabular}{ccccccccc}
\hline \hline$\rho \sigma^{3}$ & $\mu B_{0} / \epsilon$ & $\mu B / \epsilon$ & $\beta E / N-3 / 2$ & $\beta p / \rho-1$ & $\left(\rho k_{B} T K_{T}\right)^{-1}$ & $M / N \mu$ & $\chi_{L} / \rho \beta \mu^{2}$ & $\chi_{T} / \rho \beta \mu^{2}$ \\
\hline 0.10 & 5 & 5.718 & -0.253 & 0.028 & 1.083 & 0.275 & 0.592 & 0.550 \\
0.20 & 5 & 6.640 & -0.392 & 0.079 & 1.257 & 0.315 & 0.680 & 0.630 \\
0.30 & 5 & 7.837 & -0.541 & 0.156 & 1.546 & 0.365 & 0.771 & 0.729 \\
0.40 & 5 & 9.383 & -0.706 & 0.265 & 1.977 & 0.424 & 0.840 & 0.848 \\
0.50 & 5 & 11.319 & -0.890 & 0.407 & 2.606 & 0.491 & 0.847 & 0.982 \\
0.60 & 5 & 13.608 & -1.090 & 0.593 & 3.555 & 0.560 & 0.763 & 1.120 \\
0.70 & 5 & 16.138 & -1.297 & 0.842 & 5.024 & 0.623 & 0.616 & 1.246 \\
0.80 & 5 & 18.786 & -1.500 & 1.179 & 7.268 & 0.677 & 0.464 & 1.354 \\
0.90 & 5 & 21.468 & -1.685 & 1.633 & 10.596 & 0.720 & 0.339 & 1.441 \\
1.00 & 5 & 24.142 & -1.846 & 2.233 & 15.419 & 0.755 & 0.248 & 1.511 \\
\hline \hline
\end{tabular}




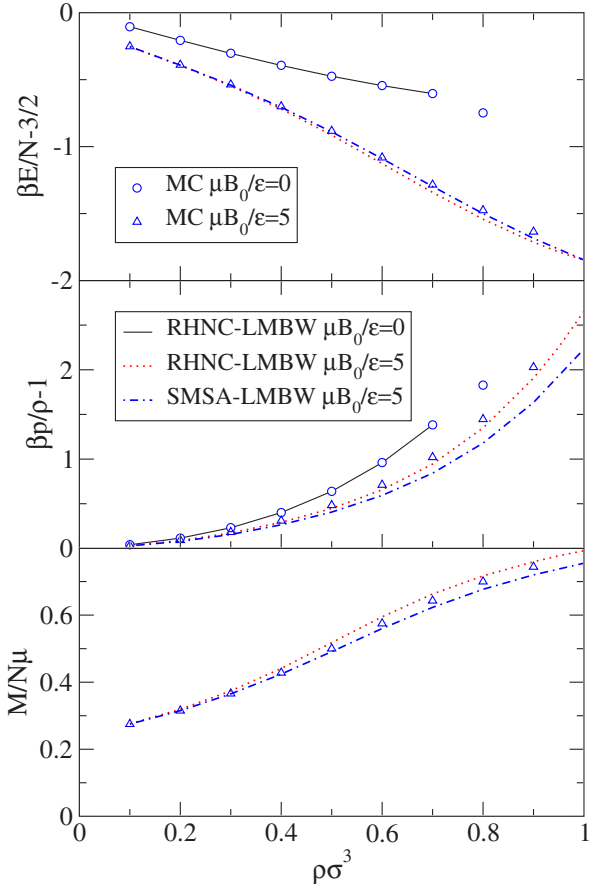

FIG. 1. (Color online) Density dependence of the excess internal energy, excess pressure, and magnetization for the $X Y$ spin fluid at $k_{B} T / \varepsilon=10$, with and without external field. Symbols denote data from Monte Carlo simulation and lines correspond to the integral equation results using the RHNC-LMBW and SMSA-LMBW combinations.

by a straightforward application of the Gram-Schmidt method [28]. Thus we can directly find for the first two generalized Chebyshev polynomials, used earlier in the expansion of the spin-spin potential $u_{s s}\left(r, \varphi_{1}, \varphi_{2}\right)$,

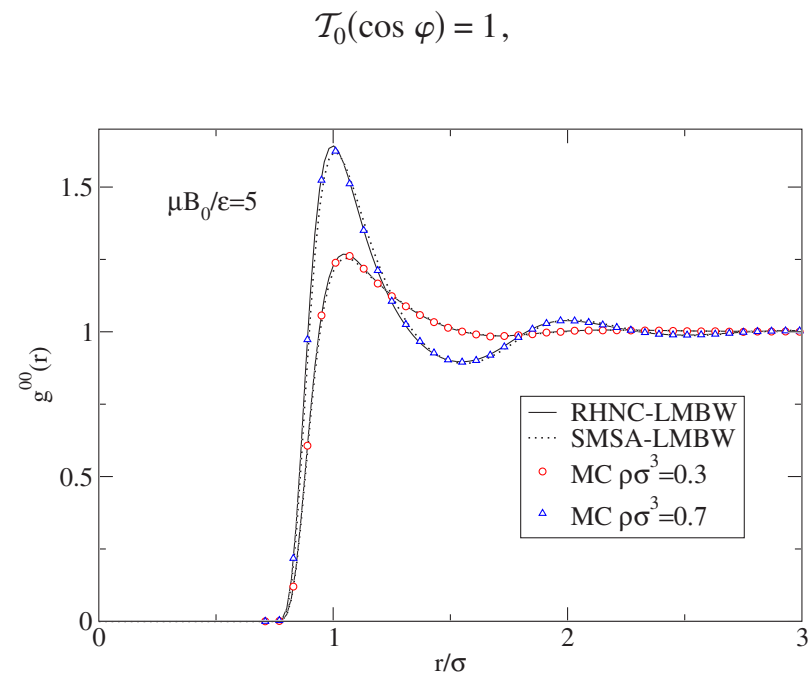

FIG. 2. (Color online) Center-to-center pair distribution function $g^{00}(r)$ calculated in the RHNC-LMBW (solid line) and SMSALMBW (dotted line) combinations along with Monte Carlo simulation data (symbols) for $\mu B_{0} / \varepsilon=5$.

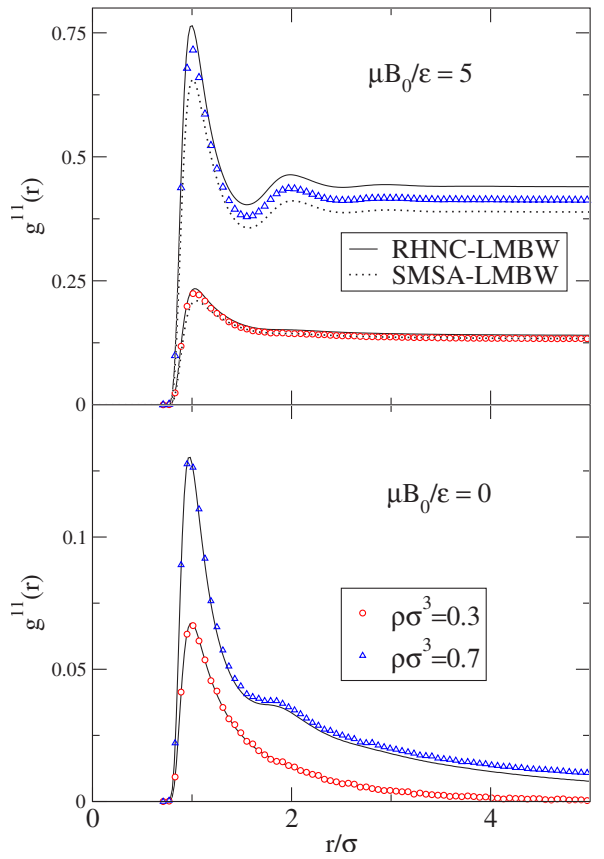

FIG. 3. (Color online) Angular coefficient $g^{11}(r)$ calculated in the RHNC-LMBW (solid lines) and SMSA-LMBW (dotted lines) combinations along with Monte Carlo simulation data (symbols); with external field (top figure) and without external field (bottom figure).

$$
\mathcal{T}_{1}(\cos \varphi)=\frac{\cos \varphi-\nu_{1}}{\left(1-2 \nu_{1}^{2}+\nu_{2}\right)^{1 / 2}} .
$$

Here and for higher-order polynomials we need the quasimoments

$$
\nu_{k} \equiv \frac{1}{\pi} \int_{0}^{\pi} d \varphi f(\varphi) \cos (k \varphi) .
$$

For the distribution

$$
f(\varphi)=\frac{e^{\beta \mu B \cos \varphi}}{I_{0}(\beta \mu B)},
$$

they are readily found to be

$$
\nu_{k}=\frac{I_{k}(\beta \mu B)}{I_{0}(\beta \mu B)} .
$$

In these expressions, $I_{j}(z)$ is the modified Bessel function of order $j$.

The simple Gram-Schmidt process, however, employs successive subtractions and so risks a progressive loss of numerical precision as higher-order polynomials are generated. This is of particular concern for accurate GaussChebyshev quadratures based on the roots of a polynomial $\mathcal{T}_{n}(x)$ with $n=10$ or larger. (In this appendix we put $x$ $\equiv \cos \varphi$.) Thus we have instead followed a robust alternative approach described by Press and Teukolsky [37]. The monic version $\pi_{m}(x) \equiv T_{m}(x) / 2^{m-1}$ for $m>0$ of the Chebyshev polynomials $T_{m}(x)$ have by construction a leading coefficient of unity and satisfy a standard recursion relation, 


$$
\begin{gathered}
\pi_{-1}(x) \equiv 0, \\
\pi_{0}(x) \equiv 1, \\
\pi_{m+1}(x)=\left(x-\alpha_{m}\right) \pi_{m}(x)-\beta_{m} \pi_{m-1}, \\
m=0,1,2, \ldots,
\end{gathered}
$$

with $\alpha_{m}=0$ and

$$
\begin{gathered}
\beta_{1}=1 / 2, \\
\beta_{m}=1 / 4, \quad m>1 .
\end{gathered}
$$

The Press-Teukolsky algorithm then uses these monic coefficients along with the $\nu_{k}$ to recursively generate a new set of coefficients $a_{m}$ and $b_{m}$ (here $a_{m}=0$ ) such that the monic polynomials $p_{m}(x)$ generated with these,

$$
\begin{gathered}
p_{-1}(x) \equiv 0, \\
p_{0}(x) \equiv 1, \\
p_{m+1}(x)=\left(x-a_{m}\right) p_{m}(x)-b_{m} p_{m-1}, \\
m=0,1,2, \ldots,
\end{gathered}
$$

are orthogonal with weight function $f(\varphi)$,

$$
\begin{aligned}
\left\langle p_{m} \mid p_{m^{\prime}}\right\rangle \equiv & \frac{1}{\pi} \int_{0}^{\pi} d \varphi f(\varphi) p_{m}(\cos \varphi) p_{m^{\prime}}(\cos \varphi)=0, \\
& \text { if } m \neq m^{\prime} .
\end{aligned}
$$

A final normalization then yields the desired generalized Chebyshev polynomials,

$$
\begin{gathered}
\mathcal{T}_{0}(x)=1, \\
\mathcal{T}_{m}(x)=\frac{p_{m}(x)}{\left[2\left\langle p_{m} \mid p_{m}\right\rangle\right]^{1 / 2}}, \quad m>0 .
\end{gathered}
$$

The algorithm further generates the roots $x_{1}, x_{2}, \ldots, x_{n}$ of $\mathcal{T}_{n}(x)=0$ and the weights $w_{j}$ for $n$-point Gauss-Chebyshev quadrature,

$$
\frac{1}{\pi} \int_{0}^{\pi} d \varphi f(\varphi) H(\cos \varphi) \approx \sum_{j=1}^{n} w_{j} H\left(x_{j}\right) .
$$

The quadrature is exact if $H(x)$ is a polynomial of degree less than or equal to $2 n-1$.

A similar procedure could now be followed for the generalization $\mathcal{U}_{m}(\cos \varphi)$ of type II Chebyshev polynomials $U_{m}(\cos \varphi)$ [28], where then $\mathcal{V}_{m}(\cos \varphi)=\sin \varphi \mathcal{U}_{m-1}(\cos \varphi)$. Here however we need the generalizations of $V_{m}(\cos \varphi)$ $=\sin m \varphi$ only through $m=4$ (in fact, through $m=3$ is adequate) and for that limited purpose the simpler GramSchmidt orthogonalization suffices. Explicitly, the first two functions, already used above for the pair potential $u_{s s}\left(r, \varphi_{1}, \varphi_{2}\right)$, are

$$
\mathcal{V}_{0}(\cos \varphi)=0
$$

$$
\mathcal{V}_{1}(\cos \varphi)=\frac{\sin \varphi}{\left(1-\nu_{2}\right)^{1 / 2}}
$$

By construction, then, these generalized Chebyshev functions $\mathcal{T}_{m}(\cos \varphi)$ and $\mathcal{V}_{m}(\cos \varphi)$ satisfy

$$
\frac{1}{\pi} \int_{0}^{\pi} d \varphi f(\varphi) \mathcal{T}_{m}(\cos \varphi) \mathcal{T}_{m^{\prime}}(\cos \varphi)= \begin{cases}1, & m=m^{\prime}=0 \\ 1 / 2, & m=m^{\prime} \neq 0 \\ 0, & m \neq m^{\prime}\end{cases}
$$

$$
\frac{1}{\pi} \int_{0}^{\pi} d \varphi f(\varphi) \mathcal{V}_{m}(\cos \varphi) \mathcal{V}_{m^{\prime}}(\cos \varphi)= \begin{cases}0, & m=m^{\prime}=0 \\ 1 / 2, & m=m^{\prime} \neq 0 \\ 0, & m \neq m^{\prime}\end{cases}
$$

$$
\frac{1}{\pi} \int_{0}^{\pi} d \varphi f(\varphi) \mathcal{T}_{m}(\cos \varphi) \mathcal{V}_{m^{\prime}}(\cos \varphi)=0
$$

Collectively, Eqs. (A12)-(A14) give rise to the orthonormalization of $\mathcal{E}_{m}(\varphi)$ expressed in Eq. (21).

For $B_{0}=0$ and $f(\varphi)=1$ so that $\nu_{k}=0$ for all $k$, the $\mathcal{T}_{m}(\cos \varphi)$ and $\mathcal{V}_{m}(\cos \varphi)$ generated in the fashion described above are of course just the standard Chebyshev functions $T_{m}(\cos \varphi)=\cos m \varphi$ and $V_{m}(\cos \varphi)=\sin m \varphi$.

\section{APPENDIX B: HELMHOLTZ FREE ENERGY}

To calculate the total free energy we use the familiar "charging" process, turning on the interactions with a parameter $\lambda, 0 \leqslant \lambda \leqslant 1$. However, as emphasized by Sullivan [38], the one-body distribution $f(\varphi)$ should remain unchanged as the interaction is turned on. Thus we will also adopt an effective external field $\mathcal{B}_{0}(\lambda)$ designed to maintain fixed the $f(\varphi)$ found by calculation, Eq. (59). Define then the partition function

$$
\begin{aligned}
Z(\lambda)= & \frac{1}{N !\left(2 \pi \Lambda^{3}\right)^{N}} \int \prod_{j=1}^{N}\left(d \mathbf{r}_{j} d \varphi_{j}\right) \exp \left[\beta \mu \mathcal{B}_{0}(\lambda) \sum_{j} \cos \varphi_{j}\right. \\
& \left.-\lambda \beta \sum_{i<j} u\left(r_{i j}, \varphi_{i}, \varphi_{j}\right)\right]
\end{aligned}
$$

where the external field is such that $\mathcal{B}_{0}(\lambda=0)=B$ and $\mathcal{B}_{0}(\lambda$ $=1)=B_{0}$. Then following the analysis of Ref. [11], we find first

$$
\begin{aligned}
\frac{\beta A}{N}= & \ln \left(\rho \Lambda^{3}\right)-1-\ln \left[I_{0}(\beta \mu B)\right]+\beta \mu\left(B-B_{0}\right) \nu_{1} \\
& +\frac{1}{2} \frac{\rho}{(2 \pi)^{2}} \int d \mathbf{r} d \varphi_{1} d \varphi_{2} f\left(\varphi_{1}\right) f\left(\varphi_{2}\right) \\
& \times \int_{0}^{1} d \lambda g\left(r, \varphi_{1}, \varphi_{2} \mid \lambda\right) \beta u\left(r, \varphi_{1}, \varphi_{2}\right) .
\end{aligned}
$$

Finally, evaluation of three of the integrals in Eq. (B2) leads to $[11]$ 


$$
\begin{aligned}
\frac{\beta A}{N}= & \ln \left(\rho \Lambda^{3}\right)-1-\ln \left[I_{0}(\beta \mu B)\right]+\beta \mu\left(B-B_{0}\right) \nu_{1}+\frac{\beta A_{1}}{N} \\
& +\frac{\beta A_{2}}{N}+\frac{\beta A_{3}}{N}
\end{aligned}
$$

with

$$
\begin{gathered}
\frac{\beta A_{1}}{N}=-\frac{1}{2} \rho \int d \mathbf{r}\left\{c_{00}(r)+\frac{1}{2} \sum_{m_{1}, m_{2}}\left[c_{m_{1} m_{2}}^{2}(r)-\gamma_{m_{1} m_{2}}^{2}(r)\right]\right\}, \\
\frac{\beta A_{2}}{N}=-\frac{1}{2 \rho} \int \frac{d \mathbf{k}}{(2 \pi)^{3}}\{\ln \operatorname{det}[\mathbf{I}+\rho \widetilde{\mathbf{H}}(k)]-\operatorname{tr}[\rho \widetilde{\mathbf{H}}(k)]\},
\end{gathered}
$$

$$
\frac{\beta A_{3}}{N}=\frac{1}{2} \rho \int d \mathbf{r} \int_{0}^{1} d \lambda \sum_{m_{1}, m_{2}} g_{m_{1} m_{2}}(r \mid \lambda) \frac{\partial b_{m_{1} m_{2}}(r \mid \lambda)}{\partial \lambda} .
$$

The final integral over $\lambda$ in Eq. (B6) cannot be evaluated in closed form and this term must be approximated. In the hypernetted-chain closure [39], it is simply neglected.

The effect of the RHNC approximation is to replace Eq. (B6) with the corresponding integral of the reference system, which is then evaluated as $A_{3}^{\text {ref }}=A^{\text {ref }}-A_{1}^{\text {ref }}-A_{2}^{\text {ref }}$. In practice, only the hard sphere system is currently known well enough for this last step; in this case, we have finally

$$
\begin{aligned}
\frac{\beta A_{\mathrm{RHNC}}}{N}= & \frac{\beta A_{\mathrm{HS}}}{N}-\ln \left[I_{0}(\beta \mu B)\right]+\beta \mu\left(B-B_{0}\right) \nu_{1}+\frac{\beta \Delta A_{1}}{N} \\
& +\frac{\beta \Delta A_{2}}{N},
\end{aligned}
$$

where $\Delta A_{k}=A_{k}-A_{k}^{\mathrm{HS}}$. For the excess hard sphere free energy $\beta A_{\mathrm{HS}}^{\mathrm{ex}} / N \equiv \beta A_{\mathrm{HS}} / N-\ln \left(\rho \Lambda^{3}\right)+1$ we use the form derived from the Carnahan-Starling equation of state [23].
[1] N. E. Frankel and C. J. Thompson, J. Phys. C 8, 3194 (1975).

[2] P. C. Hemmer and D. Imbro, Phys. Rev. A 16, 380 (1977).

[3] J. M. Tavares, M. M. Telo da Gama, P. I. C. Teixeira, J.-J. Weis, and M. J. P. Nijmeijer, Phys. Rev. E 52, 1915 (1995).

[4] J.-J. Weis, M. J. P. Nijmeijer, J. M. Tavares, and M. M. Telo da Gama, Phys. Rev. E 55, 436 (1997).

[5] A. Oukouiss and M. Baus, Phys. Rev. E 55, 7242 (1997).

[6] F. Schinagl, H. Iro, and R. Folk, Eur. Phys. J. B 8, 113 (1999).

[7] W. Fenz, R. Folk, I. M. Mryglod, and I. P. Omelyan, Phys. Rev. E 68, 061510 (2003).

[8] J. S. Høye and G. Stell, Phys. Rev. Lett. 36, 1569 (1976).

[9] T. G. Sokolovska, Physica A 253, 459 (1998).

[10] F. Lado and E. Lomba, Phys. Rev. Lett. 80, 3535 (1998).

[11] F. Lado, E. Lomba, and J.-J. Weis, Phys. Rev. E 58, 3478 (1998); 60, 2429 (1999).

[12] T. G. Sokolovska and R. O. Sokolovskii, Phys. Rev. E 59, R3819 (1999).

[13] I. P. Omelyan, I. M. Mryglod, R. Folk, and W. Fenz, Phys. Rev. E 69, 061506 (2004).

[14] I. P. Omelyan, W. Fenz, I. M. Mryglod, and R. Folk, Phys. Rev. Lett. 94, 045701 (2005).

[15] I. P. Omelyan, W. Fenz, I. M. Mryglod, and R. Folk, Phys. Rev. E 72, 031506 (2005).

[16] E. Lomba, J.-J. Weis, N. G. Almarza, F. Bresme, and G. Stell, Phys. Rev. E 49, 5169 (1994).

[17] M. J. P. Nijmeijer and J.-J. Weis, Phys. Rev. Lett. 75, 2887 (1995); Phys. Rev. E 53, 591 (1996).

[18] M. J. P. Nijmeijer and J.-J. Weis, in Annual Review of Computational Physics IV, edited by D. Stauffer (World Scientific, Singapore, 1996).

[19] A. L. Ferreira and W. Korneta, Phys. Rev. E 57, 3107 (1998).

[20] M. Blume, V. J. Emery, and R. B. Griffiths, Phys. Rev. A 4, 1071 (1971).
[21] A. Maciołek, M. Krech, and S. Dietrich, Phys. Rev. E 69, 036117 (2004).

[22] D. J. Tulimieri, J. Yoon, and M. H. W. Chan, Phys. Rev. Lett. 82, 121 (1999).

[23] J.-P. Hansen and I. R. McDonald, Theory of Simple Liquids (Academic, London, 1986).

[24] D. Henderson, in Fundamentals of Inhomogeneous Fluids, edited by D. Henderson (Dekker, New York, 1992), Chap. 4.

[25] R. Lovett, C. Y. Mou, and F. P. Buff, J. Chem. Phys. 65, 570 (1976).

[26] M. S. Wertheim, J. Chem. Phys. 65, 2377 (1976).

[27] So named by M. S. Green [J. Chem. Phys. 33, 1403 (1960); 39, 1367 (1963)] because of the resemblance of the first diagram in its density expansion to a Wheatstone bridge.

[28] See, for example, G. Arfken, Mathematical Methods for Physicists (Academic, Orlando, 1985).

[29] F. Lado, Phys. Rev. A 8, 2548 (1973).

[30] Y. Rosenfeld and N. W. Ashcroft, Phys. Rev. A 20, 1208 (1979).

[31] F. Lado, Phys. Lett. 89A, 196 (1982).

[32] L. Verlet and J.-J. Weis, Phys. Rev. A 5, 939 (1972).

[33] D. Henderson and E. W. Grundke, J. Chem. Phys. 63, 601 (1975).

[34] See, for example, E. O. Brigham, The Fast Fourier Transform (Prentice-Hall, Englewood Cliffs, NJ, 1974), Chap. 10.

[35] E. Lomba, C. Martín, and N. G. Almarza, Mol. Phys. 101, 1667 (2003).

[36] G. Zerah and J.-P. Hansen, J. Chem. Phys. 84, 2336 (1986).

[37] W. H. Press and S. A. Teukolsky, Comput. Phys. 4, 423 (1990)

[38] D. E. Sullivan, Phys. Rev. A 25, 1669 (1982).

[39] T. Morita and K. Hiroike, Prog. Theor. Phys. 23, 1003 (1960). 